\title{
Physicochemical properties of tamarillo (Solanum betaceum Cav.) hydrocolloid fractions
}

\begin{abstract}
Tamarillo (Solanum betaceum Cav.) is an underutilised fruit in Malaysia. The fruit, however, contains good proportions of soluble fibre, protein, starch, anthocyanins and carotenoids. Amongst the fruits, only tamarillo mesocarp contains both polar (anthocyanins) and non-polar (carotenoids) pigments. The ability to retain both polar and non-polar pigments in the mesocarp could be related to the unique properties of its hydrocolloids. To understand the pigmenthydrocolloid interaction in the fruit, information on the physicochemical characteristics of the hydrocolloids is required. Therefore, hydrocolloids from the anthocyanin-rich seed mucilage fraction of the tamarillo and its carotenoid-rich pulp fraction were extracted and characterised. Water and $1 \%$ citric acid were used to extract the seed mucilage hydrocolloid while $72 \%$ ethanol and $20 \mathrm{mM}$ HEPES buffer were used for pulp hydrocolloid extraction. Seed mucilage hydrocolloid was primarily composed of arabinogalactan protein-associated pectin whereas pulp hydrocolloid was composed of hemicellulosic polysaccharides with some naturally interacting proteins and neutral polysaccharides.
\end{abstract}

Keyword: Extraction; Physicochemical properties; Tamarillo; Hydrocolloid; Pigments 